\title{
CLINICAL DIFFERENCES OF CRYPTOGENIC ISCHEMIC STROKE IN YOUNG PATIENTS WITH AND WITHOUT PATENT FORAMEN OVALE
}

\section{Introduction}

Patent foramen ovale (PFO) is a frequent finding in young patients with cryptogenic ischemic stroke (IS). However, little is known about clinical characteristics of PFO-related stroke. We compared acute circumstances and symptomatology between cryptogenic IS with and without PFO.

\section{Methods}

We included patients with cryptogenic IS at age 18-49 enrolled in the multicenter SECRETO study (NCTO1934725) who underwent standardized transesophageal echocardiography with Valsalva maneuver and contrast to examine presence of PFO (any degree of shunt). We compared demographics, premonitory symptoms, mode of symptom onset, neurological and cardiac symptoms (chest pain/palpitation), and occurrence of Valsalva inducing activity or heavy physical exertion within 2 hours prior to stroke in patients with and without PFO.
\end{abstract}

O. Nerg1, N. Martinez-Majander² , S. Saeed3, P. Jäkälä1, H. Numminen4, P. Ylikotila5, J. Huhtakangas6, U. Junttola ${ }^{6}$, N. Yesilot7, M. Zedde8, C. Fonseca9, U. Waje-Andreassen ${ }^{10}$, P. Redfors ${ }^{11}$, B. von Sarnowski12, R.O. Roine5, T. Tatlisumak'11, J. Sinisalo13, E. Gerdts3, J. Putaala on behalf of the SECRETO Consortium. ${ }_{1}^{1}$ Kuopio University Hospital, Neurology, Kuopio, Finland. ${ }^{2}$ Helsinki University Hospital, Neurology, Helsinki, Finland. 3 Haukeland University Hospital, Cardiology, Bergen, Norway. ${ }^{4}$ Tampere University Hospital, Neurology, Tampere, Finland. 5 Turku University Hospital, Division of Clinical Neurosciences, Turku, Finland. ${ }^{6}$ Oulu University Hospital, Neurology, Oulu, Finland. 7Istanbul University, Faculty of Medicine, Istanbul, Turkey. ${ }^{8}$ Azienda Unità Sanitaria Locale - IRCCS, Neuromotor Physiology, Reggio Emilia, Italy. ${ }^{9}$ Hospital de Santa Maria, Neurology, Lisboa, Portugal. ${ }^{10}$ Haukeland University Hospital, Neurology, Bergen, Norway. ${ }^{11}$ Sahlgrenska University Hospital, Neurology, Gothenburg, Sweden. ${ }^{12}$ University Medicine Greifswald, Neurology, Greifswald, Germany. ${ }^{33 H e l s i n k i}$ Norway. ${ }^{11}$ Sahlgrenska University Hospital, Neurology, Gothenburg, Sweden. ${ }^{12}$ University Medicine Greifs
University Hospital, Cardiology, Helsinki, Finland.

\section{Results}

Of the 201 patients (mean age 38.6+/-8.o years; $57.1 \%$ men; median admission NIH Stroke Scale [NIHSS] score 2, range 0-20; $21.7 \%$ treated with iv-thrombolysis, 6.9\% with thrombectomy), 109 (54.2\%) had PFO. PFO patients' symptom onset was more often abrupt than gradual compared to non-PFO patients (79.4\% vs. $64.1 \%$; $\mathrm{P}=0.002)$, aphasia more common $(36.7 \%$ vs. $19.6 \% ; \mathrm{P}=0.008)$, but nausea/vomiting (16.5\% vs. $29.3 \% ; \mathrm{P}=0.030)$ and headache less frequent (31.2\% vs. 46.7\%; P=0.024). PFO patients had lower NIHSS score (median 1 vs. $3 ; \mathrm{P}<0.001)$.

In logistic regression adjusted for age and sex, abrupt mode of stroke onset (OR 2.11, 95\% CI 1.06-4.21), aphasia (OR 2.89, 95\% CI 1.36-6.13), and lower NIHSS score (OR 0.88, 95\% CI 0.80-0.95) were independently associated with PFO-related IS.

Table 1. Demographic and physiologic parameters

\begin{tabular}{|c|c|c|c|}
\hline & PFO - & PFO + & p-value \\
\hline Age (mean) & $\begin{array}{l}37.7 y \\
{[36.0-39.5]}\end{array}$ & $\begin{array}{l}40.2 y \\
{[38.8-41.5]}\end{array}$ & 0.337 \\
\hline Sex & $\begin{array}{l}\text { male } 50(54.3 \%) \\
\text { female } 42(45.7 \%)\end{array}$ & $\begin{array}{l}\text { male } 65(59.6 \%) \\
\text { female } 44(40.7 \%)\end{array}$ & 0.451 \\
\hline $\begin{array}{l}\text { Treated with i.v } \\
\text { thrombolysis }\end{array}$ & $23(11.3 \%)$ & $21(10.3 \%)$ & 0.370 \\
\hline $\begin{array}{l}\text { Treated with } \\
\text { thrombectomy }\end{array}$ & $8(3.9 \%)$ & $6(3.0 \%)$ & 0.399 \\
\hline $\begin{array}{l}\text { Mean NIHSS on } \\
\text { admission }\end{array}$ & $\begin{array}{l}4.5 \\
{[3.56-5.44]}\end{array}$ & $\begin{array}{l}2.4 \\
{[1.73-3.04]}\end{array}$ & 0.001 \\
\hline $\begin{array}{l}\text { Systolic BP on } \\
\text { admission }\end{array}$ & $\begin{array}{l}145.2 \mathrm{mmHg} \\
{[139.9-150.5]}\end{array}$ & $\begin{array}{l}140.0 \mathrm{mmHg} \\
{[136.0-144.0]}\end{array}$ & 0.077 \\
\hline $\begin{array}{l}\text { Diastolic BP on } \\
\text { admission }\end{array}$ & $\begin{array}{l}87.8 \mathrm{mmHg} \\
{[84.6-91.1]}\end{array}$ & $\begin{array}{l}84.3 \mathrm{mmHg} \\
{[81.9-86.8]}\end{array}$ & 0.076 \\
\hline $\begin{array}{l}\text { Heartrate on } \\
\text { admission }\end{array}$ & $\begin{array}{l}79.7 \mathrm{~b} / \mathrm{min} \\
{[76.5-83.0]}\end{array}$ & $\begin{array}{l}77.0 \mathrm{~b} / \mathrm{min} \\
{[74.0-80.0]}\end{array}$ & 0.148 \\
\hline $\begin{array}{l}\text { Temperature on } \\
\text { admission }\end{array}$ & $\begin{array}{l}36.7^{\circ} \mathrm{C} \\
{[36.6-36.9]}\end{array}$ & $\begin{array}{l}36.7^{\circ} \mathrm{C} \\
{[36.6-36.8]}\end{array}$ & 0.398 \\
\hline $\begin{array}{l}\text { Systolic BP } \\
\text { following morning }\end{array}$ & $\begin{array}{l}132.2 \mathrm{mmHg} \\
{[128.0-136.5]}\end{array}$ & $\begin{array}{l}126.8 \mathrm{mmHg} \\
{[123.1-130.6]}\end{array}$ & 0.045 \\
\hline $\begin{array}{l}\text { Diastolic BP } \\
\text { following morning }\end{array}$ & $\begin{array}{l}79.0 \mathrm{mmHg} \\
{[76.0-82.0]}\end{array}$ & $\begin{array}{l}75.2 \mathrm{mmHg} \\
{[72.8-77.5]}\end{array}$ & 0.057 \\
\hline $\begin{array}{l}\text { Heartrate following } \\
\text { morning }\end{array}$ & $\begin{array}{l}68.5 \mathrm{bmin} \\
{[66.1-70.9]}\end{array}$ & $\begin{array}{l}68.5 \mathrm{bmin} \\
{[66.3-70.8]}\end{array}$ & 0.975 \\
\hline Prior TIA & $5(5.4 \%)$ & $4(3.7 \%)$ & 0.658 \\
\hline Wake up stroke & $21(22.8 \%)$ & $24(22.2 \%)$ & 0.282 \\
\hline
\end{tabular}

Table 2. Symptomatology

\begin{tabular}{|c|c|c|c|}
\hline & PFO - & PFO + & p-value \\
\hline $\begin{array}{l}\text { Mode of stroke onset - } \\
\text { gradual }\end{array}$ & $14(15.2 \%)$ & $2(1.9 \%)$ & 0.565 \\
\hline $\begin{array}{l}\text { Mode of stroke onset - } \\
\text { abrupt }\end{array}$ & $59(64.1 \%)$ & $85(79.4 \%)$ & 0.029 \\
\hline Aphasia & $18(19.6 \%)$ & $40(36.7 \%)$ & 0.008 \\
\hline Nausea or vomiting & $27(29.3 \%)$ & $18(16.5 \%)$ & 0.030 \\
\hline Headache & $43(46.7 \%)$ & $34(31.2 \%)$ & 0.024 \\
\hline Decreased conciousness & $6(6.5 \%)$ & $9(8.3 \%)$ & 0.641 \\
\hline Dysarthria & $33(36.3 \%)$ & 37 (33.9\%) & 0.629 \\
\hline Hemianopsia & $18(19.6 \%)$ & $16(14.7 \%)$ & 0.440 \\
\hline Gaze deviation & $6(6.5 \%)$ & $5(4.6 \%)$ & 0.462 \\
\hline Ataxia & $33(35.9 \%)$ & $28(25.9 \%)$ & 0.307 \\
\hline Diplopia & $10(10.9 \%)$ & $13(11.9 \%)$ & 0.815 \\
\hline Vertigo & $43(46.7 \%)$ & $41(38.0 \%)$ & 0.317 \\
\hline Motor paresis & $63(68.5 \%)$ & $65(59.6 \%)$ & 0.312 \\
\hline Sensory deficit & $60(65.2 \%)$ & $58(53.7 \%)$ & 0.160 \\
\hline Dysphagia & $7(7.7 \%)$ & $4(3.7 \%)$ & 0.310 \\
\hline Chest pain & $1(1.1 \%)$ & $0(0.0 \%)$ & 0.273 \\
\hline Palpitation & $4(4.4 \%)$ & $1(0.9 \%)$ & 0.198 \\
\hline $\begin{array}{l}\text { Valsalva } 1-2 \mathrm{~h} \text { prior to } \\
\text { stroke }\end{array}$ & $10(13.9 \%)$ & $16(17.6 \%)$ & 0.522 \\
\hline $\begin{array}{l}\text { Heavy physical exertion } \\
\text { during } 1 \mathrm{~h} \text { prior to stroke }\end{array}$ & $3(1.5 \%)$ & $3(1.5 \%)$ & 0.783 \\
\hline $\begin{array}{l}\text { Heavy physical exertion } \\
\text { during } 2 \mathrm{~h} \text { prior stroke }\end{array}$ & $1(1.1 \%)$ & $2(1.9 \%)$ & 0.694 \\
\hline
\end{tabular}

\section{Conclusions}

Cryptogenic IS patients with PFO had more often sudden symptom onset suggestive of embolic etiology than non-PFO-patients, albeit they had milder symptoms on admission. 